\title{
Virtual Restoration of Ancient Chinese Paintings Using Color Contrast Enhancement and Lacuna Texture Synthesis
}

\author{
Soo-Chang Pei, Fellow, IEEE, Yi-Chong Zeng, and Ching-Hua Chang
}

\begin{abstract}
This work presents a novel algorithm using color contrast enhancement and lacuna texture synthesis is proposed for the virtual restoration of ancient Chinese paintings. Color contrast enhancement based on saturation and de-saturation is performed in the $u^{\prime} v^{\prime} Y$ color space, to change the saturation value in the chromaticity diagram, and adaptive histogram equalization then is adopted to adjust the luminance component. Additionally, this work presents a new patching method using the Markov Random Field (MRF) model of texture synthesis. Eliminating undesirable aged painting patterns, such as stains, crevices, and artifacts, and then filling the lacuna regions with the appropriate textures is simple and efficient. The synthesization procedure integrates three key approaches, weighted mask, annular scan and auxiliary, with neighborhood searching. These approaches can maintain a complete shape and prevent edge disconnection in the final results. Moreover, the boundary between original and synthesized paintings is seamless, and unable to distinguish in which the undesirable pattern appears.
\end{abstract}

Index Terms-Color contrast enhancement, lacuna texture synthesis, Markov random field, virtual restoration.

\section{INTRODUCTION}

$\mathbf{T}$ HE uses of digital processing for image improvement recently has received considerable interest and has broad applications in medical imagery, remote sensing, digital multimedia, image transmission, and so on. The digital processing includes four types of image manipulation processes, namely: image compression, image enhancement, image restoration and geometrical image modification [1]. The main objective of image enhancement techniques is to process an image, and producing a new image that is more suitable than the original for certain specific applications [2]. No general unifying theory and image quality standard exists that can suggest design criteria for image enhancement processing. Moreover, processing image enhancement for restoration and reconstruction becomes even more difficult when dealing with ancient paintings.

Numerous techniques exist for image enhancement. Some of these techniques focus on emphasizing the local image inten-

Manuscript received February 21, 2003; revised October 6, 2003. This work was supported by the National Science Council, R.O.C., under Contract NSC91-2219-E-002-044 and by the Ministry of Education under Contract 89-E-FA06-2-4. The associate editor coordinating the review of this manuscript and approving it for publication was Dr. Henri Maitre.

S.-C. Pei is with the Department of Electrical Engineering, National Taiwan University, Taipei 10617, Taiwan, R.O.C. (e-mail: pei@cc.ee.ntu.edu.tw).

Y.-C. Zeng and C.-H. Chang are with the Graduate Institute of Communication Engineering, National Taiwan University, Taipei 10617, Taiwan, R. O. C. (e-mail: d9942010@ee.ntu.edu.tw; r90942087@ms90.ntu.edu.tw).

Digital Object Identifier 10.1109/TIP.2003.821347 sity or color variations to enhance perceptual visibility. Accordingly, most of these techniques work in some color spaces where the intensity and chromaticity components can be separated and adopted using the human visual perception model.

This study describes the hybrid method in the XYZ and $\mathrm{L} \mathrm{u}^{\prime} \mathrm{v}^{\prime}$ color spaces of the CIE primary color coordinate system [3], which enables the combination of the enhancement operations. Owing to the nonuniform character of the commonly used xy chromaticity diagram, we propose selecting the more uniform $\mathrm{u}^{\prime} \mathrm{v}^{\prime}$ chromaticity diagram. For many short lines in the xy chromaticity diagram joining a pair of points, which represent two colors with perceptual color difference of the same magnitude, these identical color differences should be represented by lines of equal length. In fact, these lines are much longer than the average length toward the green part of the spectral locus, and much shorter toward the violet part. Since, the ratio of the longest to the shortest line in the $\mathrm{u}^{\prime} \mathrm{v}^{\prime}$ chromaticity diagram is only around four to one, instead of around twenty to one in the xy chromaticity diagram [3], color mixing performance based on color distance is better in the $\mathrm{u}^{\prime} \mathrm{v}^{\prime}$ chromaticity diagram. Lucchese and Mitra originally proposed the nonlinear filtering and enhancement techniques for color images in the xy chromaticity diagram [4], [5]. Fig. 1 adopts the saturation and de-saturation concept using the center of gravity law for color mixture [3], [5] in the uniform $\mathrm{u}^{\prime} \mathrm{v}^{\prime}$ chromaticity domain, and combines them with adaptive histogram modification [6] to adjust the luminance component $\mathrm{Y}$ to a suitable brightness level. The final image is enhanced with more appealing color in the brighter region and more brightness contrast in the darker region. Sharpness also is increased, and visible details of the resultant image.

Texture synthesis involves synthesizing numerous textures from original samples, and their structure characteristic is similar to sample structure. Efros [7] and Wei [8] use the Markov random field/Gibbs sampling, to model texture, and perform synthesis with probability sampling. Additionally, matching sample texture appearance using a pyramid-model, Heeger and Bergen developed an algorithm that obtained better-synthesized results of random textures [9]. Moreover, the approach of DeBonet preserved the cross-scale dependencies of the sample texture, and moreover works better on structured textures [10].

The texture synthesis technique inspires us to repair ancient paintings, eliminating undesirable patterns and filling it with texture. The undesirable aged painting patterns displayed in Fig. 2 generally include stains, crevices and artifacts (words 

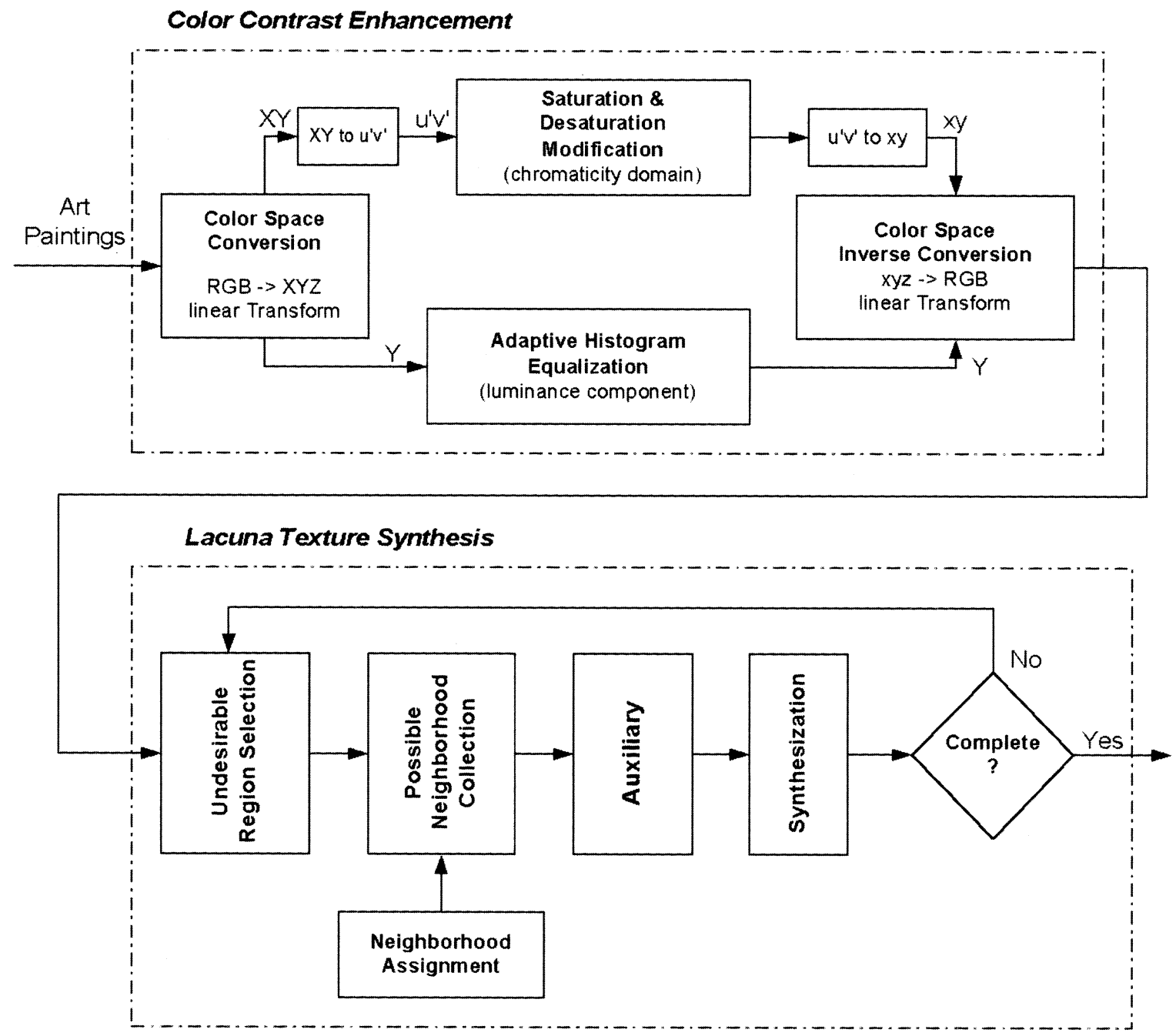

Fig. 1. Procedures of hybrid color contrast enhancement and lacuna texture synthesis.

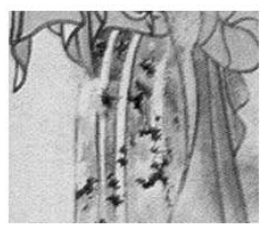

(a)

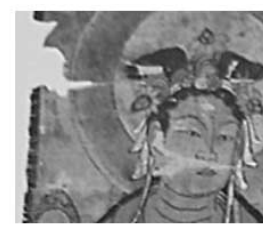

(b)

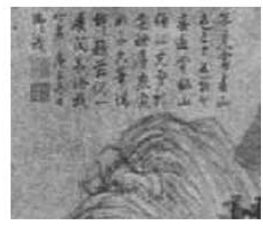

(c)
Fig. 2. Three types of undesirable patterns, (a) stains, (b) crevices, and (c) words and signet artifacts.

or signets), and so on. This study proposes a new texture synthesis algorithm using the weighted mask, annular scan and auxiliary to fix damage on paintings of various ages. The algorithm successfully restores the digital imageries of ancient Chinese paintings, and the experimental results demonstrate its superiority to previous techniques.

The remainder of this paper is organized as follows. First, Section II presents the hybrid color contrast enhancement method. Subsequently, Section III describes the proposed texture synthesis method. The experimental results and discussion then are presented separately in Sections IV and V. Finally, conclusions are given in Section VI.

\section{HYBRID COLOR CONTRAST ENHANCEMENT}

Hybrid color contrast enhancement includes color enhancement in the chromatic domain and brightness enhancement in the luminance component. These two enhancements are not independent, when the Y component is adjusted slightly to control the illumination level [5]. The Y component also is modified using adaptive histogram equalization following color enhancement in the $\mathrm{u}^{\prime} \mathrm{v}^{\prime}$ component, because this method of order processing achieves better image quality, and Fig. 1 illustrates the experimental procedure.

Rather than working in the RGB color space, operations are performed in the $\mathrm{u}^{\prime} \mathrm{v}^{\prime}$ chromaticity domain of CIE L $\mathrm{u}^{\prime} \mathrm{v}^{\prime}$ color space. The linear transformations between the RGB and CIE $\mathrm{XYZ}$ color spaces are given by

$$
\left(\begin{array}{l}
X \\
Y \\
Z
\end{array}\right)=\left(\begin{array}{lll}
0.49000 & 0.31000 & 0.20000 \\
0.17697 & 0.81240 & 0.01063 \\
0.00000 & 0.01000 & 0.99000
\end{array}\right)\left(\begin{array}{c}
R \\
G \\
B
\end{array}\right)
$$

The chromaticity values $\mathrm{u}^{\prime}$ and $\mathrm{v}^{\prime}$ then are obtained as below,

$$
\begin{aligned}
\mathrm{u}^{\prime} & =\frac{4 X}{X+15 Y+3 Z} \\
\mathrm{v}^{\prime} & =\frac{9 Y}{X+15 Y+3 Z}
\end{aligned}
$$




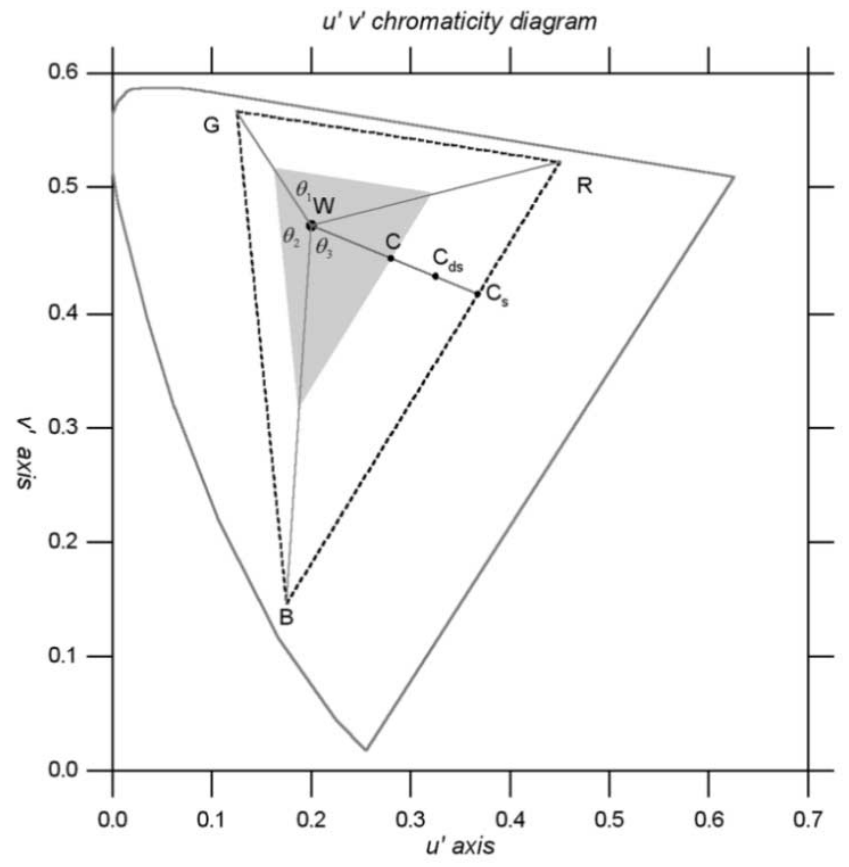

Fig. 3. The dotted triangle represents color gamut of standard screen, and the blue curve represents the spectral locus.

Fig. 3 represents the $\mathrm{u}^{\prime} \mathrm{v}^{\prime}$ chromatic plane. The three vertices of the color triangle are located at $\mathrm{R}\left(\mathrm{u}^{\prime}=0.4507, \mathrm{v}^{\prime}=0.5229\right)$, $\mathrm{G}\left(\mathrm{u}^{\prime}=0.1250, \mathrm{v}^{\prime}=0.5625\right), \mathrm{B}\left(\mathrm{u}^{\prime}=0.1754, \mathrm{v}^{\prime}=0.1579\right)$ and D65 white point [3] is located at $\mathrm{W}\left(\mathrm{u}^{\prime}=0.1978, \mathrm{v}^{\prime}=\right.$ $0.4683)$. Since $\mathrm{u}^{\prime}$ and $\mathrm{v}^{\prime}$ represent chromatic components and $\mathrm{Y}$ contains the brightness information, it is reasonable to adopt $\left(\mathrm{u}^{\prime}, \mathrm{v}^{\prime}, \mathrm{Y}\right)$ as the working space. Thus this approach enhances the chromatic and luminance parts, respectively.

\section{A. Color Enhancement}

1) Achromatic Triangle: All color pixels in the gamut triangle except the achromatic region can be saturated and de-saturated. Since the points around the white point are not very colorful, enhancing these color pixels is meaningless. Thus this region near the white point is treated as the achromatic region. The achromatic region proposed in [5] is a circle around the white point, but some discontinuous color pixels are resulted due to over-saturation around the long lines, $\overline{\mathrm{WR}}, \overline{\mathrm{WG}}$, and $\overline{\mathrm{WB}}$ in Fig. 3. Instead of the achromatic circle, the small similar triangle with half the three sides of the gamut triangle is chosen as the achromatic triangle. Discontinuous color pixels are avoided when choosing the proposed achromatic triangle. No saturation and de-saturation operations work for the pixels inside the achromatic region. However, two main processes are involved for the pixels outside the achromatic region, namely saturation and de-saturation.

2) Saturation: All color pixels of an image are discretely distributed in a $u^{\prime} v^{\prime}$ diagram. As illustrated in Fig. 3, three types of angular ranges, $\theta_{1}, \theta_{2}$ and $\theta_{3}$, are composed by $\overline{\mathrm{WR}}$, $\overline{\mathrm{WG}}$, and $\overline{\mathrm{WB}}$, respectively. Assume that a color image pixel $\mathrm{C}$ and the white point $\mathrm{W}$, are represented as $\left(\mathrm{u}^{\prime}, \mathrm{v}^{\prime}, \mathrm{Y}\right)$ and $\left(\mathrm{u}_{\mathrm{w}}^{\prime}, \mathrm{v}_{\mathrm{w}}^{\prime}, \mathrm{Y}_{\mathrm{w}}\right)$ respectively. Stretch the line between the white point, $\mathrm{W}$, and point $\mathrm{C}$ outside the triangle. Specifying $\mathrm{C}$ in one

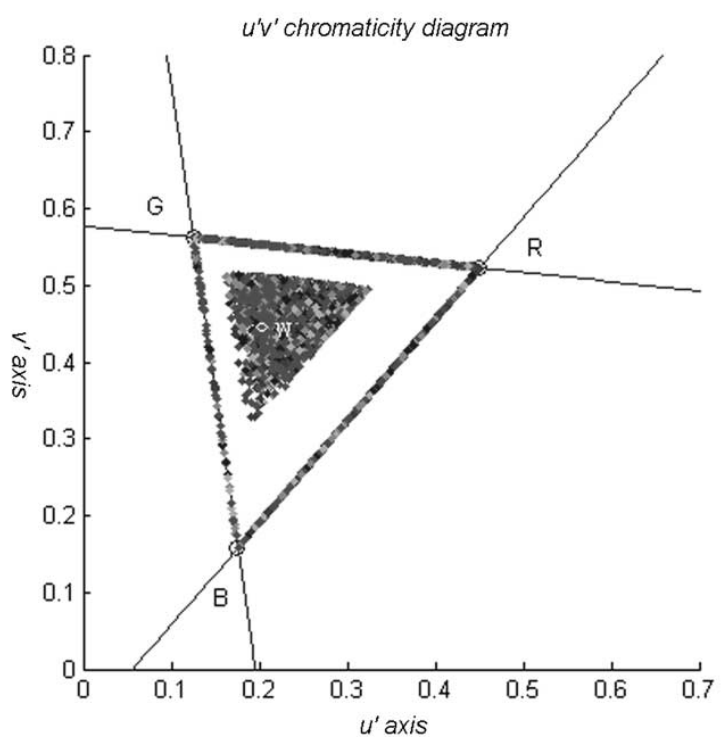

(a)

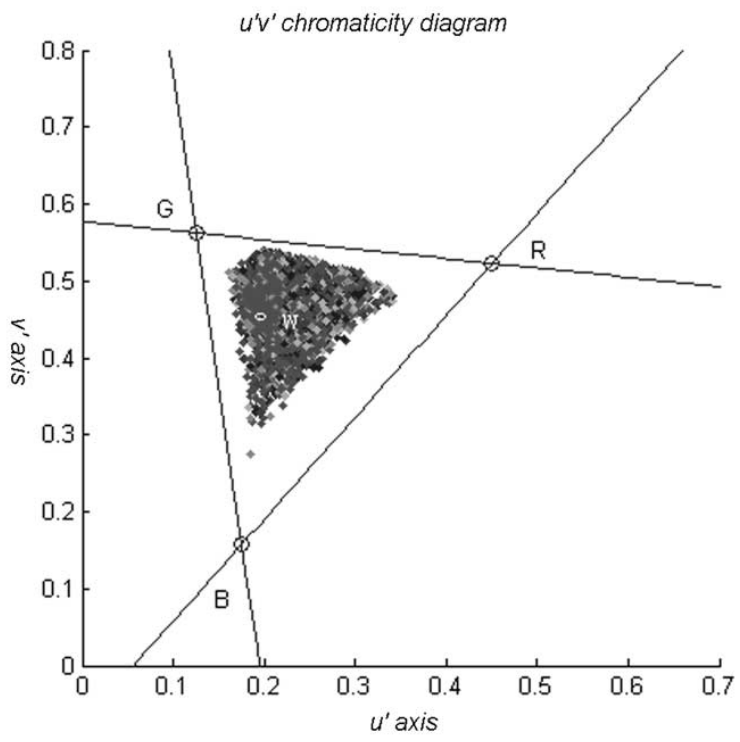

(b)

Fig. 4. (a) All the color pixels except those inside the achromatic triangle are moved to the gamut triangle boundaries during the saturation procedure. (b) All of the color pixels, except those inside the achromatic triangle, are de-saturated within the gamut triangle.

of three kinds of angular range can identify which line $\overline{\mathrm{WC}}$ intersects, namely $\overline{\mathrm{GR}}, \overline{\mathrm{BG}}$ or $\overline{\mathrm{RB}}$. Fig. 4(a) shows that every color pixel, except the points inside the achromatic triangle, is removed from their original location to the boundaries of the gamut triangle. However, the hue of each color pixel is fixed.

The removed color pixel can be represented as $\mathrm{C}_{\mathrm{s}}\left(\mathrm{u}_{\mathrm{s}}^{\prime}, \mathrm{v}_{\mathrm{s}}^{\prime}, \mathrm{Y}\right)$. Since this pixel is maximally saturated, the values of the $\mathrm{u}_{\mathrm{s}}^{\prime} \mathrm{v}_{\mathrm{s}}^{\prime}$ chromaticity components are maximized as displayed on the monitor. However, the maximally saturated image appears unnatural, meaning that it is better to proceed with de-saturation.

3) De-Saturation: Following saturation, all color pixels outside the achromatic triangle are moved to the gamut triangle 


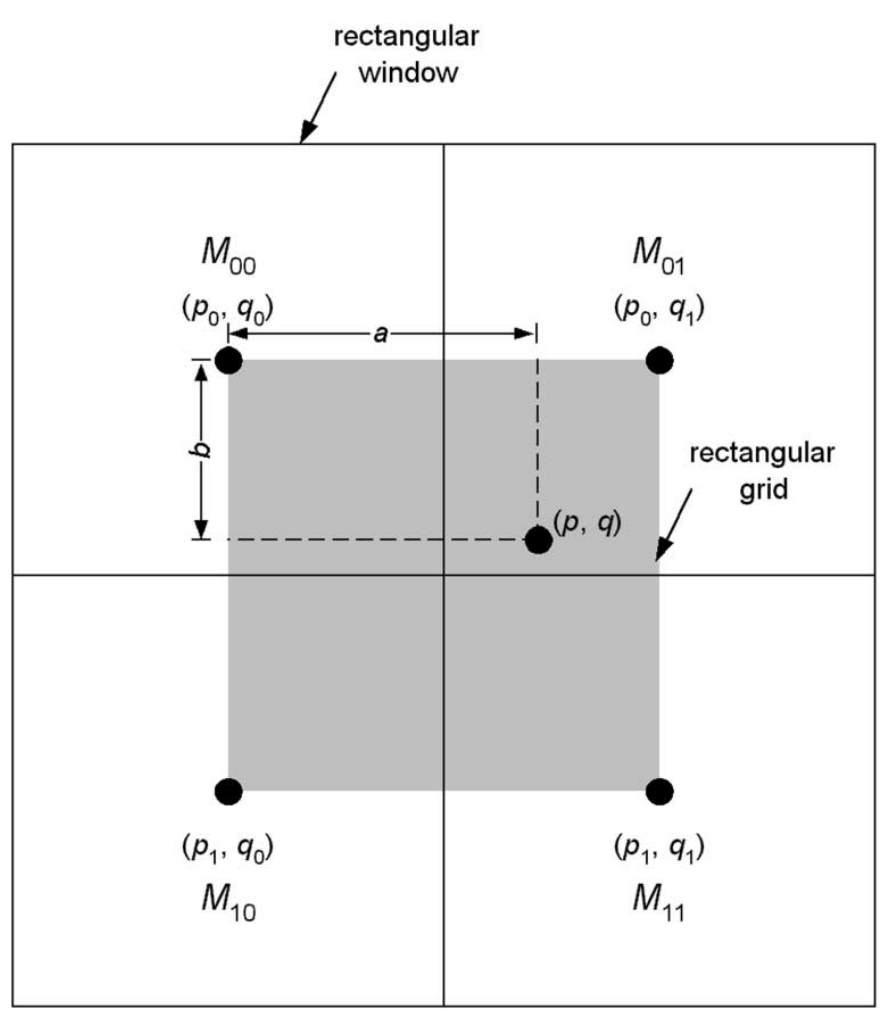

Fig. 5. $M_{00}, M_{01}, M_{10}$ and $M_{11}$ are transfer functions for histogram modification mapping. The new value at $(p, q)$ is evaluated by bilinear interpolation.

boundaries. The color pixels of the natural image are nonuniformly distributed within the triangle. De-saturation must be proceeded properly. De-saturation is implemented by using the Center of Gravity Law of color mixing [3], [5]. This approach assumes that the chromaticity coordinates of $\mathrm{C}_{\mathrm{w}}$ and $\mathrm{C}_{\mathrm{S}}$ are defined as $\left(\mathrm{u}_{\mathrm{w}}^{\prime}, \mathrm{v}_{\mathrm{w}}^{\prime}, \mathrm{Y}_{\mathrm{w}}\right)$ and $\left(\mathrm{u}_{\mathrm{s}}^{\prime}, \mathrm{v}_{\mathrm{s}}^{\prime}, \mathrm{Y}\right)$ respectively, then $\mathrm{C}_{\mathrm{ds}}\left(\mathrm{u}_{\mathrm{ds}}^{\prime}, \mathrm{v}_{\mathrm{ds}}^{\prime}, \mathrm{Y}_{\mathrm{ds}}\right)$ results from two color mixtures, as follows,

$$
u_{d s}^{\prime}=\frac{u_{s}^{\prime} \frac{Y}{v_{s}^{\prime}}+u_{w}^{\prime} \frac{Y_{w}}{v_{w}^{\prime}}}{\frac{Y}{v_{s}^{\prime}}+\frac{Y_{w}^{w}}{v_{w}^{w}}}, \quad v_{d s}^{\prime}=\frac{Y+Y_{w}}{\frac{Y}{v_{s}^{\prime}}+\frac{Y_{w}}{v_{w}^{\prime}}}
$$

$\mathrm{Y}_{\mathrm{w}}$ is chosen as $\mathrm{Y}_{\mathrm{w}}=k \overline{\mathrm{Y}}$ where $k$ is a positive factor for controlling the luminance level and $\bar{Y}$ is the mean luminance of all pixels. In the virtual restoration conducted here, $k$ is assigned a positive value, so that the picture will appear brighter. Experimentally, the chosen value of $k$ varies from 0 to 0.5 , the larger the factor $k$ is chosen, the more the color pixels are de-saturated. Fig. 4(b) shows that the color pixels on the boundaries of the gamut triangle are de-saturated using the (3). Finally, the luminance of the whole image is slightly increased by the $\mathrm{Y}_{\mathrm{ds}}=\mathrm{Y}_{\mathrm{w}}+\mathrm{Y}$. This proposed color contrast enhancement makes the pictures appear more colorful, and consequently can increase image sharpness.

\section{B. Brightness Enhancement}

Since Y represents the luminance component, it is enhanced by the well-known adaptive histogram equalization (AHE) [6]. Fig. 5 shows how a picture can be divided into several nonoverlapping rectangular regions. AHE applies histogram modification to each pixel based on the histogram of pixels within a moving window neighborhood. Histogram modification mappings, $M_{00}(i), M_{10}(i), M_{01}(i)$ and $M_{11}(i)$ are generated at four neighboring grid points at $\left(p_{0}, q_{0}\right),\left(p_{1}, q_{0}\right),\left(p_{0}, q_{1}\right)$ and $\left(p_{1}, q_{1}\right)$. The mapping applied at $(p, q)$ is obtained using the following bilinear interpolation.

$$
\begin{array}{r}
M(i)=(1-a)\left[(1-b) M_{00}(i)+b M_{10}(i)\right] \\
+a\left[(1-b) M_{01}(i)+b M_{11}(i)\right]
\end{array}
$$

, where $a=\left(q-q_{0}\right) /\left(q_{1}-q_{0}\right), b=\left(p-p_{0}\right) /\left(p_{1}-p_{0}\right)$, and $i$ denotes the gray value at $(p, q)$. For the boundary pixels, the pixels within the image can be reflected outside the borders. Thus, the boundary pixels do not require any special handling. For experiment of Fig. 10, the original image, rectangular grid and rectangular window sizes are $1859 \times 1315,201 \times 201$ and $201 \times 201$, respectively. If the rectangular window size is too small, the image will appear unnatural since too much local details on small regions are enhanced in too small an area. However, if the rectangular window size is large, then the effects on the achromatic region will be less obvious. As a result, experimental size choice depends on the image features.

Both of the luminance and chromaticity components already have been adjusted. First, xy components are obtained from (5), as follows,

$$
\mathrm{x}=\frac{9 \mathrm{u}^{\prime}}{6 \mathrm{u}^{\prime}-16 \mathrm{v}^{\prime}+12}, \quad \mathrm{y}=\frac{4 \mathrm{u}^{\prime}}{6 \mathrm{u}^{\prime}-16 \mathrm{v}^{\prime}+12}
$$

The $\mathrm{X}, \mathrm{Y}$ and $\mathrm{Z}$ are re-distributed using the following formulas,

$$
\begin{aligned}
\mathrm{Z} & =1-\mathrm{x}-\mathrm{y}, \quad \mathrm{X}+\mathrm{Y}+\mathrm{Z}=\frac{\mathrm{Y}}{\mathrm{y}}, \\
\mathrm{X} & =\mathrm{x}(\mathrm{X}+\mathrm{Y}+\mathrm{Z}) \\
\mathrm{Z} & =\mathrm{z}(\mathrm{X}+\mathrm{Y}+\mathrm{Z})
\end{aligned}
$$

Thus, $\mathrm{X}, \mathrm{Y}$ and $\mathrm{Z}$ can be converted to the $\mathrm{R}, \mathrm{G}$ and $\mathrm{B}$ color space using the inverse transformation of (1).

\section{LACUNA TeXture Synthesis}

This section presents a novel four-phase texture synthesis algorithm, including: neighborhood assignment, possible neighborhood collection, auxiliary and synthesization. The algorithm is designed to eliminate undesirable patterns in aged paintings and fill the lacuna regions with appropriate textures. The procedure of lacuna texture synthesis is illustrated in Fig. 1.

\section{A. Neighborhood Assignment}

Similar to the method of Efros, this study adopts a square-window neighborhood with width $L$ for patching the damaged paintings. Two types of fields exist in the neighborhood of $W S(p)$ (the central pixel of which moves in the $\boldsymbol{S}$ area in Fig. 6), those are normal field $W S_{n}(p)$ and synthesized field $W S_{s}(p)$. The characteristics of both fields must satisfy the following,

$$
\begin{gathered}
W S_{n}(p) \cup W S_{s}(p)=W S(p) \\
W S_{n}(p) \cap W S_{s}(p)=0 \\
A_{k}^{W S}(p)= \begin{cases}0, & \text { if } k t h \text { pixel belongs to } W S_{s}(p) \\
1, & \text { otherwise }\end{cases}
\end{gathered}
$$

where $p$ denotes the position $(x, y)$ of the central synthesized pixel and the variable $A_{k}^{W S}(p)$ represents the attribution of the $k$ th pixel in $W S(p)$. We define the attribution of synthesized 


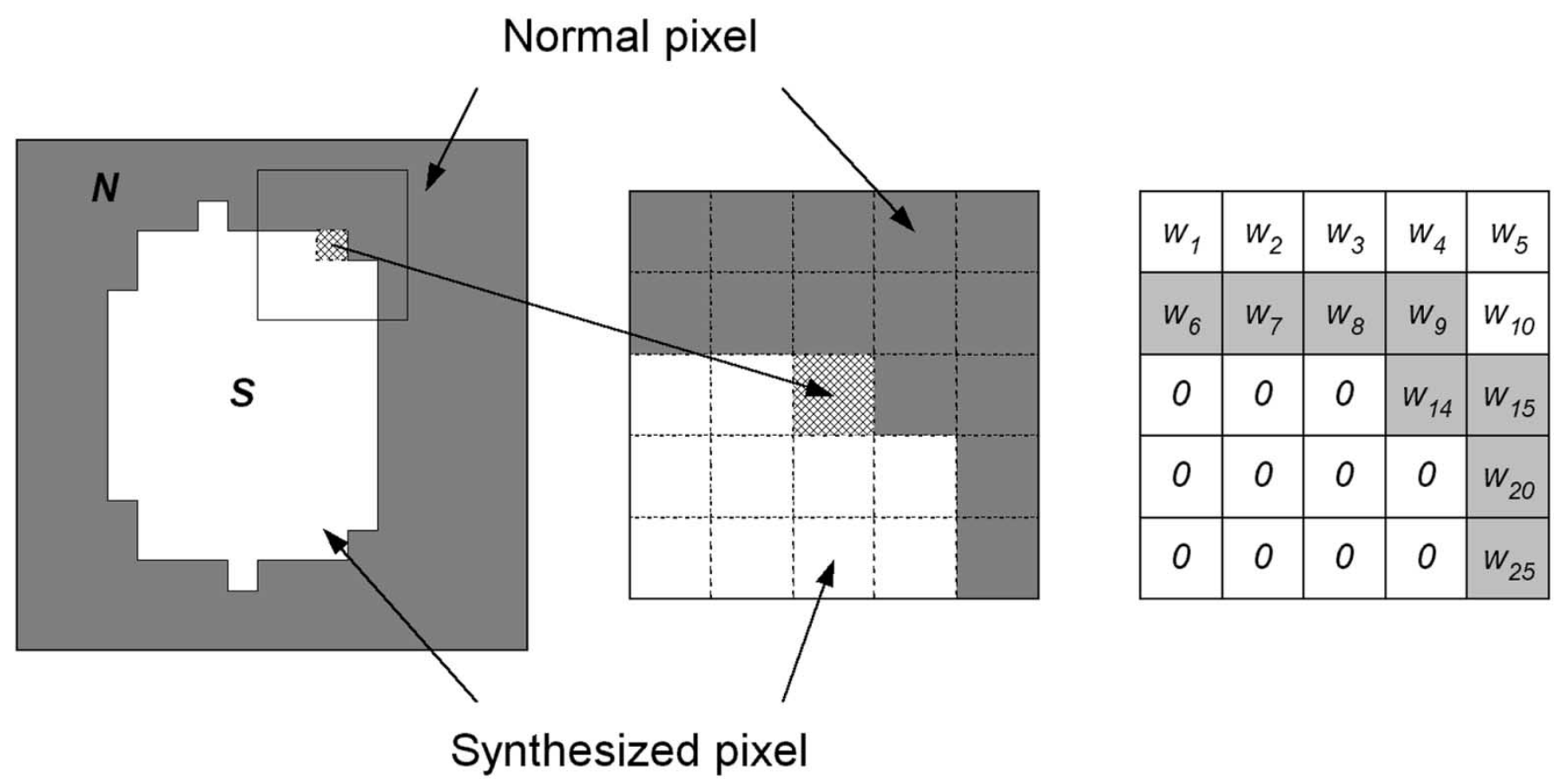

Fig. 6. Corrupt image (leftmost) with a $5 \times 5$ square neighborhood (middle) and weighted mask (rightmost).

pixel as $A_{k}^{W S}(p)=0$; meanwhile, $A_{k}^{W S}(p)=1$ indicates the normal pixel.

Fig. 6 shows a square neighborhood extracted from the leftmost corrupt image. The dark gray area indicates the normal filed, and the white area indicates the synthesized field. For a size $L \times L$ square neighborhood, all pixels except for the central synthesized pixel, that is $L^{2}-1$ pixels, are required for neighborhood searching.

\section{B. Possible Neighborhood Collection}

During this phase, the possible neighborhood is collected from the sample. Notably, the strategy does not resemble that employed in previous studies [8]-[12], in which neighborhoods are collected from the pre-prepared sample. The substitute sample here consists of pixels, are excluded from the corrupt image. It is deemed as one of self-similarity characteristic. In [11], Brooks et al. implemented texture editing based on self-similarity, and acquires the sample from the synthesized image itself just as with the method presented here.

A square-window possible neighborhood $W N\left(p_{i}\right)$, the central pixel of which moves in the $N$ area in Fig. 6, comes from the corrupt image. Two types of field exist, namely normal field $W N_{n}\left(p_{i}\right)$ and synthesized field $W N_{s}\left(p_{i}\right)$, in the $i$ th $W N\left(p_{i}\right)$ at position $p_{i}$. The attribution is formulated as,

$$
A_{k}^{W N}\left(p_{i}\right)= \begin{cases}0, & \text { if } k t h \text { pixel belongs to } W N_{s}\left(p_{i}\right) \\ 1, & \text { otherwise }\end{cases}
$$

, where the variable $A_{k}^{W N}\left(p_{i}\right)$ represents the $k$ th pixel's attribution in $W N\left(p_{i}\right)$. This study defines the attribution of the synthesized pixel as $A_{k}^{W N}\left(p_{i}\right)=0$; meanwhile, $A_{k}^{W N}\left(p_{i}\right)=1$ indicates the normal pixel.

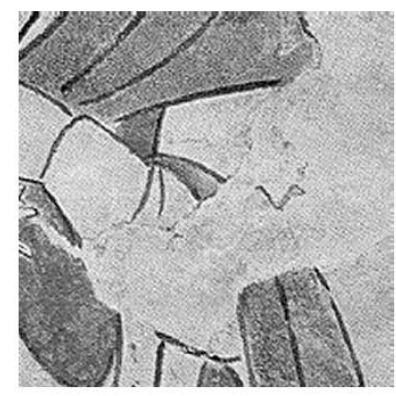

(a)

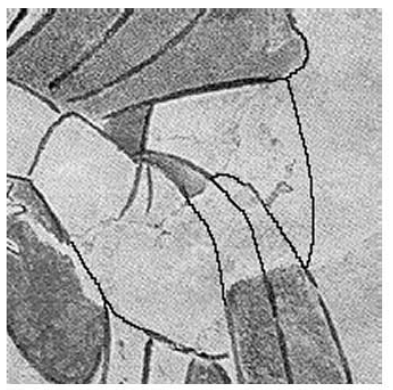

(b)
Fig. 7. For the corrupt image (leftmost), it adds the auxiliary lines drawn (rightmost).

\section{Auxiliary}

Unfortunately, various types of damage, e.g. stain, crevice, artifacts (words or signets), have different effects on ancient paintings. Here, we propose a simple approach for overcoming the effects by adding some auxiliaries to the corrupt paintings.

Fig. 7(a) represents an illustrative example representing a shawl draped on the left arm. The boundary between the arm and the background had vanished as well as one part of the shawl was peeled off. The corrupt region covers many different textures, including arms, sleeves, shawl, clothes and background. A lack of auxiliary can easily cause failure synthesization, and Fig. 17(b) shows an experimental result. Therefore, drawing the distinct boundary between each of the different textures being synthesized is essential, and Fig. 7(b) shows the image with auxiliary lines drawn.

\section{Synthesization}

Two important synthesizing rules exist for identifying corrupt regions and then determining the extent to which they should be 


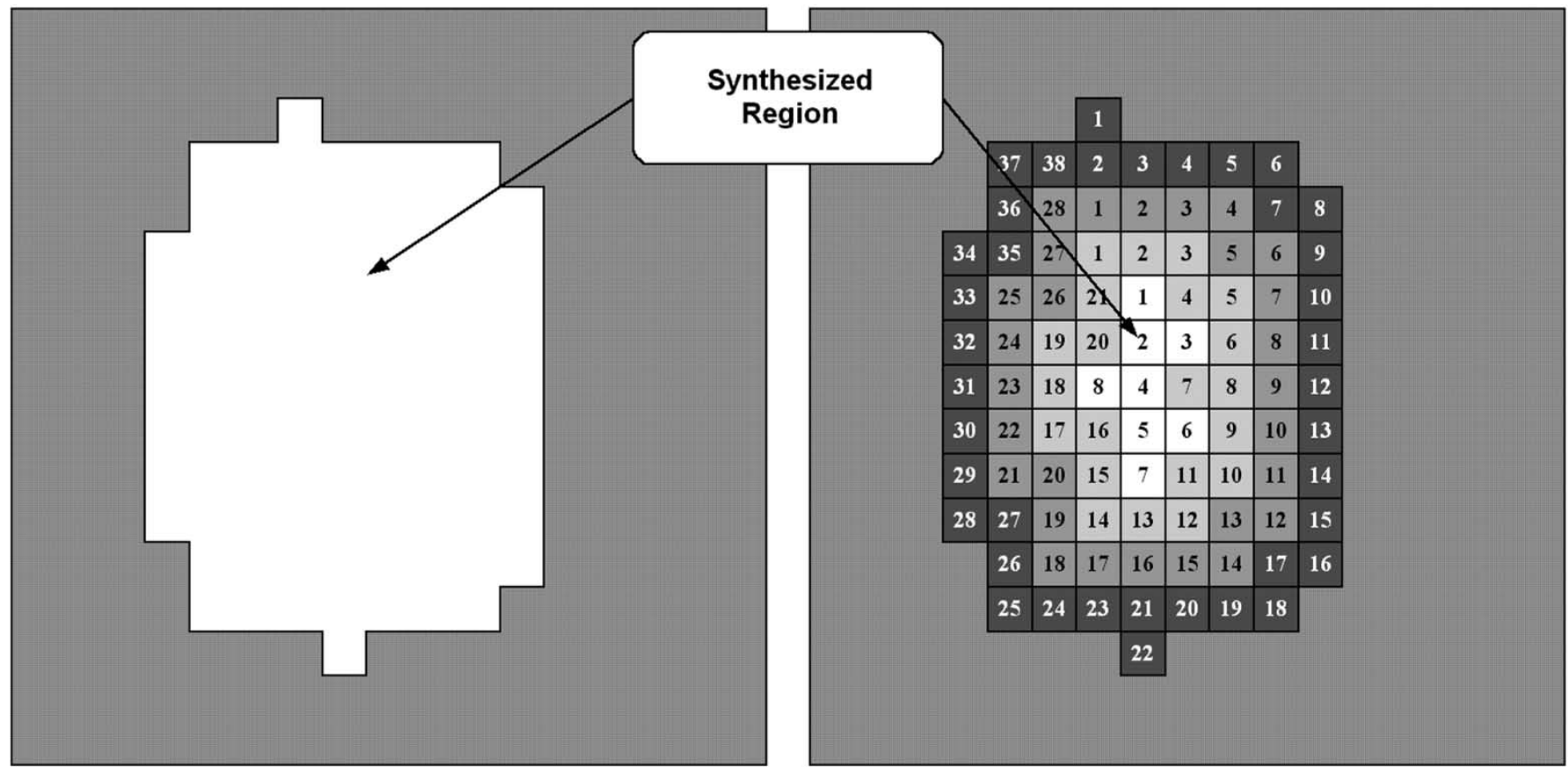

Fig. 8. Corrupt image and its annular scan.

marked. Both rules are related to pattern recognition and image segmentation. To simplify the procedure, this study manually marks the region, then decides where the auxiliary should be added.

Unlike pervious works [10], this study applies the annular scan rather than the raster scan. The annular scan as implied in the name treads along the outside boundary of the patched region. It assigns one pixel width from outside the boundary, and then gradually progresses toward the inside boundary. Fig. 8 illustrates the diagram of the annular scan, in which each square denotes a synthesized pixel and it runs along the smaller number pixels to larger number pixels in the same boundary squares. The boundaries then shrink from dark to bright squares.

This study constructs a neighborhood $W S(p)$ whose center is a synthesized pixel, and then searches a neighborhood $W N\left(p_{i}\right)$ most similar to $W S(p)$. Consequently, the central pixel value of $W S(p)$ is replaced by the corresponding one in $W N\left(p_{i}\right)$. The perceptual distance $d(S, N)$ measures the similarity between neighborhoods $S$ and $N$. Unlike the conventional square error distance measurement, the algorithm uses a weighted distance as follow,

$$
d\left(W S(p), W N\left(p_{i}\right)\right)=\sum_{k} w_{k}\left[W S_{k}(p)-W N_{k}\left(p_{i}\right)\right]^{2}
$$

, where $W S_{k}(p)$ and $W N_{k}\left(p_{i}\right)$ represent the $k$ th pixel value in $W S(p)$ and $W N\left(p_{i}\right)$, respectively. Moreover, variable $w_{k}$ is a normalized weight to emphasize the edge connection. Two types of pixels in particular are defined in the normal field to be weighted mask, namely, the outbound pixel and the boundary pixel. The former does not belong to any of eight-connectivity of the synthesized pixels, $W_{1}, W_{2}, W_{3}, W_{4}, W_{5}$ and $W_{10}$ in Fig. 6. On the contrary, the later is one of the eight-connectivity of the synthesized pixels, $W_{6}, W_{7}, W_{8}, W_{9}, W_{14}, W_{15}, W_{20}$ and $W_{25}$ in Fig. 6. The use of weighted mask can avoid the shape incompleteness following synthesis. The formulas are defined as follow,

$$
\begin{aligned}
w_{k} & = \begin{cases}\frac{1}{A} W_{k}^{B}, & \text { if } W S_{k}(p) \text { belongs to } \\
\text { boundary pixel }\end{cases} \\
\frac{1}{A} W_{k}^{O}, & \text { elsewise }
\end{aligned}
$$

, where $W_{k}^{B}$ and $W_{k}^{O}$ denote the weighted values of the boundary and outbound pixels, respectively, and the normalization factor $A$ summarizes all of the weighted values.

From (14), since the normal pixels in $W N\left(p_{i}\right)$ perfectly match those in $W S(p)$, the measured distance is correct; however, if the normal field $W N_{n}\left(p_{i}\right)$ does not match $W S_{n}(p)$, then an infinite distance value is assigned

$$
\begin{aligned}
& d\left(W S(p), W N\left(p_{i}\right)\right) \\
& = \begin{cases}d\left(W S(p), W N\left(p_{i}\right)\right), & \text { if } \sum_{k} A_{k}^{W S}(p) \cdot A_{k}^{W N}\left(p_{i}\right) \\
& =\sum_{k} A_{k}^{W S}(p) \\
\infty, & \text { otherwise }\end{cases}
\end{aligned}
$$

\section{EXPERIMENT RESULTS}

\section{A. Color Contrast Enhancement}

As mentioned previously, this study focuses on the restoration of ancient Chinese paintings, with the chosen test images being some representative artworks from the Tang Dynasty to the Ming Dynasty [12]. Several pictures are sourced from the database of articles and images contained in the periodicals of the National Palace Museum, while the others, which are digitized by scanner, come from paintings books published by the National Place Museum. 


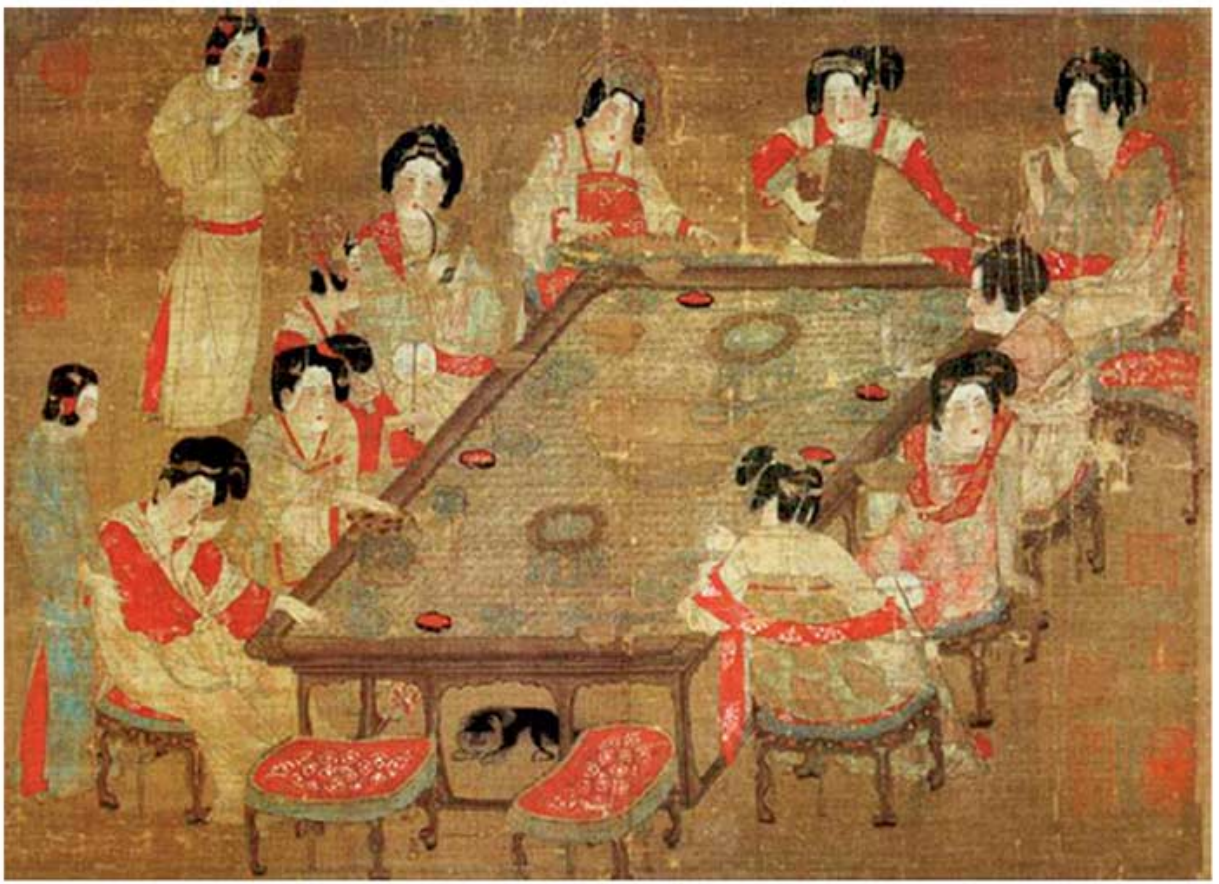

(a)

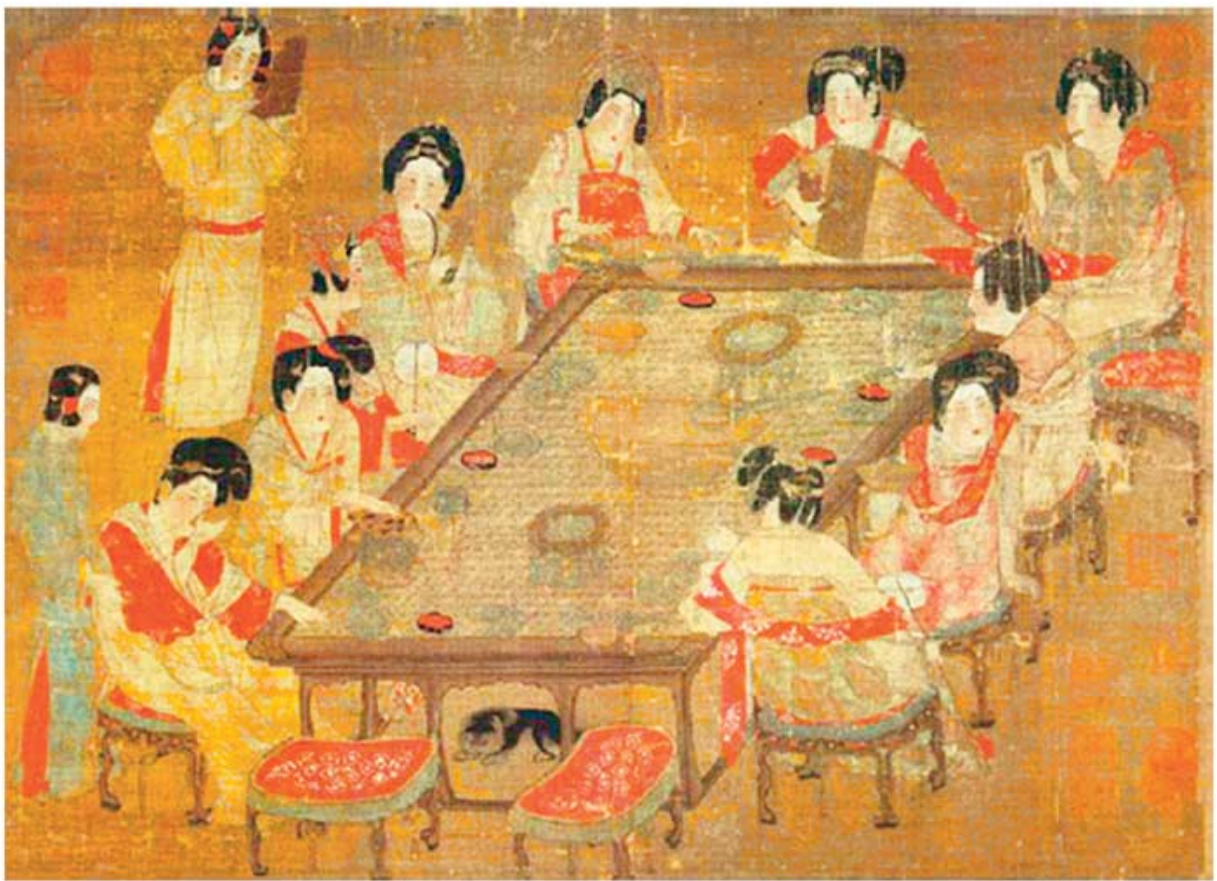

(b)

Fig. 9. (a) Original image of "A Palace Concert" in the Tang Dynasty and (b) color contrast enhancement by saturation and de-saturation $(k=0.3)$.

Three main experiments are performed. One experiment uses color contrast enhancement only, another combines color contrast enhancement with brightness contrast enhancement, and the last one uses the hybrid method without AHE. First, the test image uses color contrast enhancement is shown in Fig. 9(a). It displays a traditional Chinese painting of Tang Dynasty ladies named "A Palace Concert." In Fig. 9(b), it displays the two steps involves in the color contrast enhancement, and shows how this process makes the ladies appear more vivid. Factor $k$ can be dynamically altered depending on image appearance.

The saturation and de-saturation operations enhance the image not only by changing gloomy regions, but also by increasing image sharpness. Fig. 10(a) shows a classical painting from the Sung Dynasty named "Wind in Pines among Myriad Valleys." Importantly, the Y component regarded as gray level can be applied along with other brightness enhancement. 


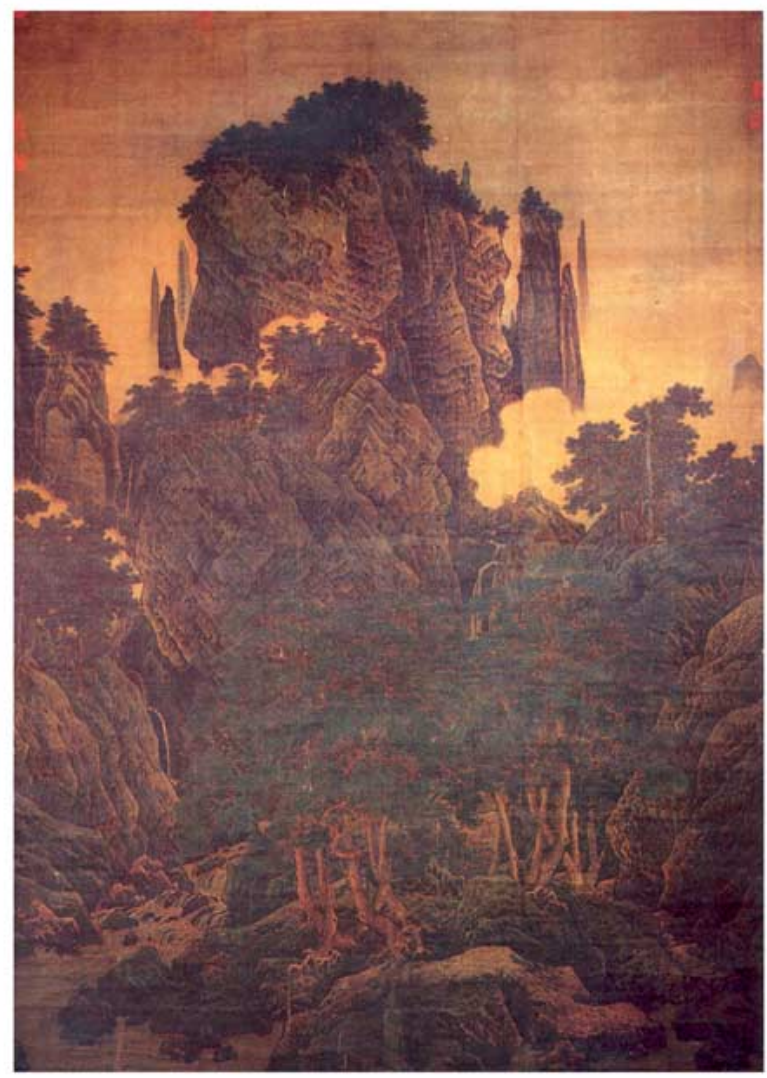

(a)

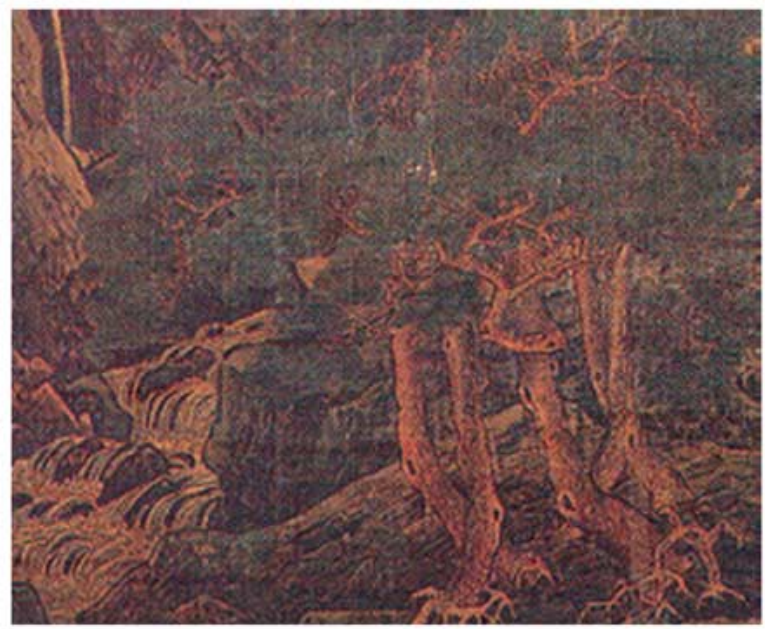

(c)

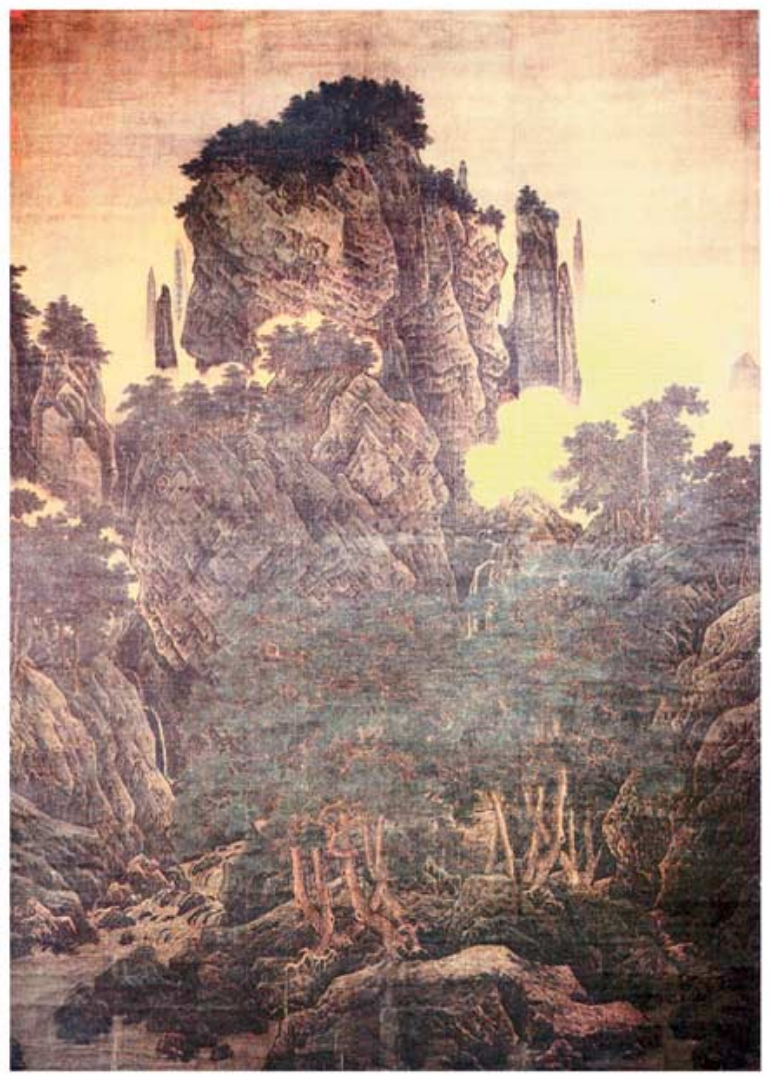

(b)

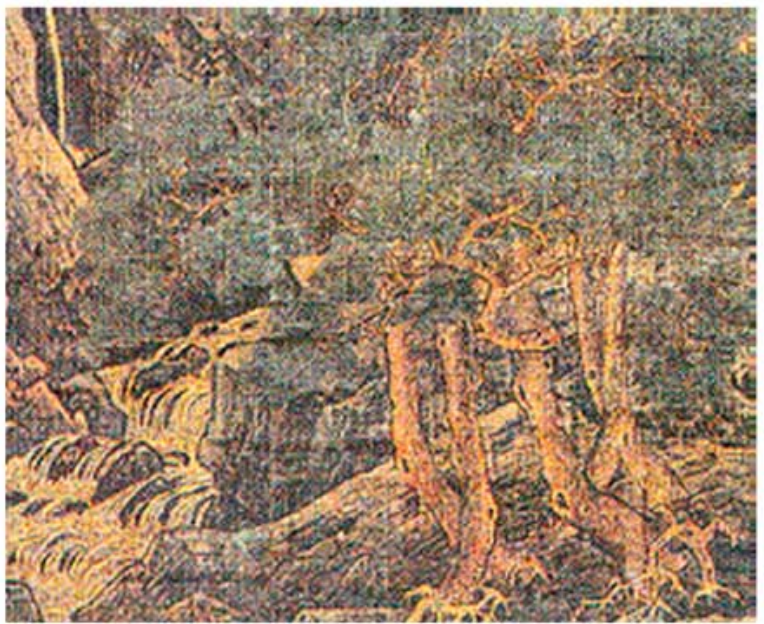

(d)

Fig. 10. (a) Original image of "Wind in Pines Among Myriad Valleys" with size $1859 \times 1315$; (b) enhancement by saturation, de-saturation $(k=0.1)$ and AHE (rectangular window size is $201 \times 201$ ); (c) partial region of (a) includes the branches of the pine in the painting; and (d) details of the same region are enhanced by AHE after color contrast enhancement $(k=0.1$, rectangular window size is $201 \times 201)$.

Numerous unseen details, such as the branches of the pines, are revealed when the color contrast enhancement is combined with the adaptive histogram modification. Fig. 10(b) is processed by AHE following color contrast enhancement. Figs. 10(c) and 10(d) show the same enlarged regions as shown in Figs. 10(a) and 10(b), respectively. The restored image reveals more details, such as the texture of the rock, than the original image, and moreover this is achieved without significant color loss. Fig. 11(a) illustrates a painting from the Yuan Dynasty called "Autumn Colors on the Ch'iao and Hua Mountains." Moreover, Fig. 11(b) shows the same image enhanced by saturation and de-saturation operations in color contrast enhancement. Finally, 


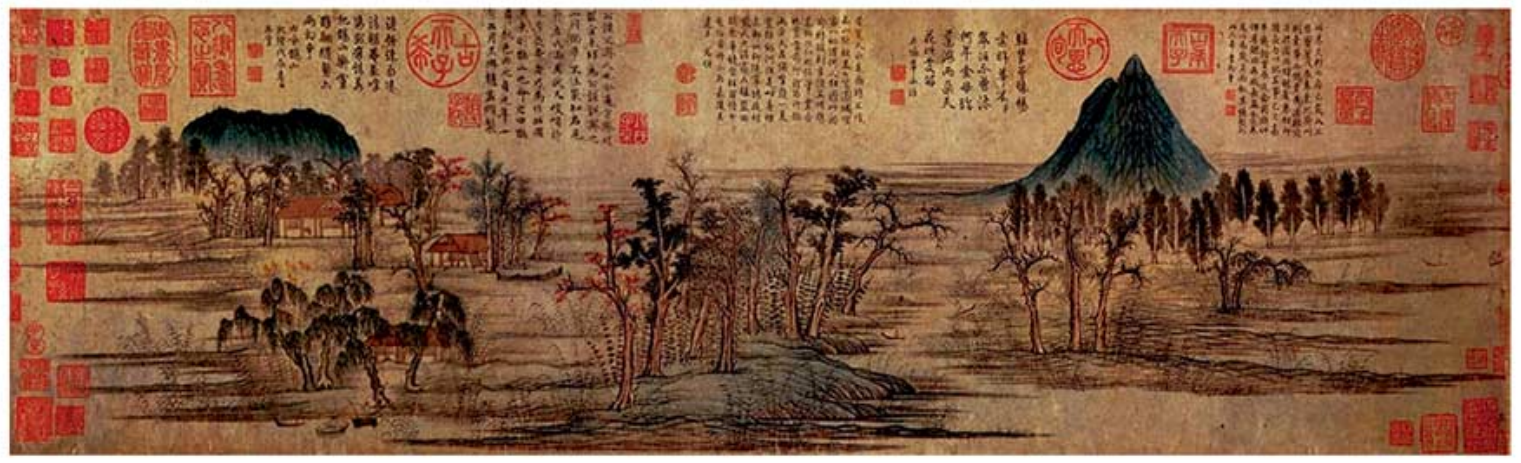

(a)

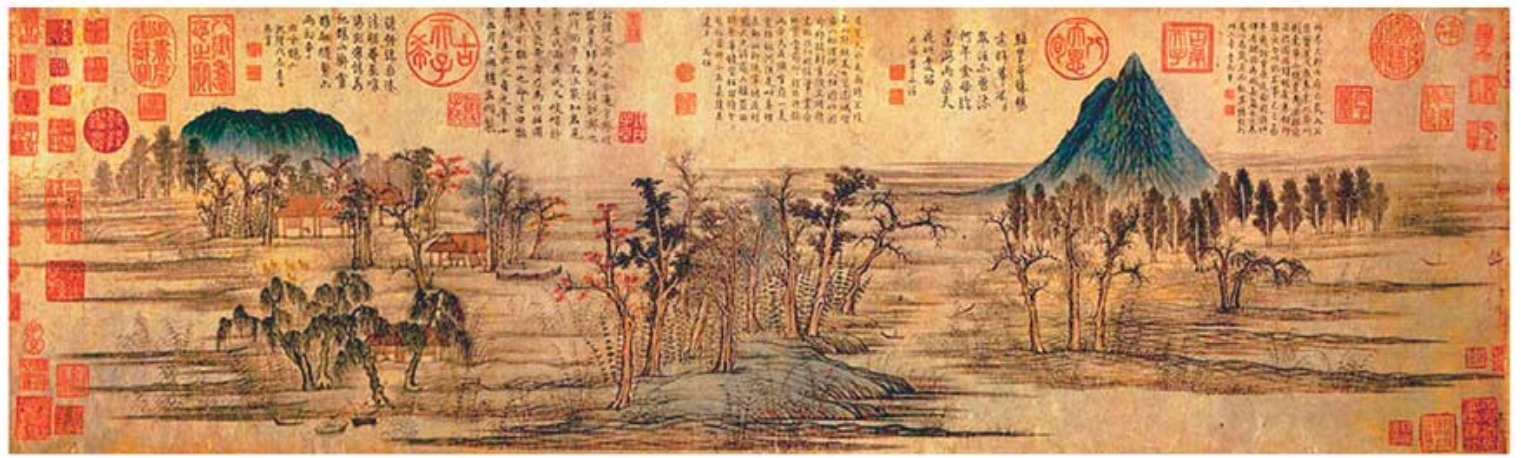

(b)

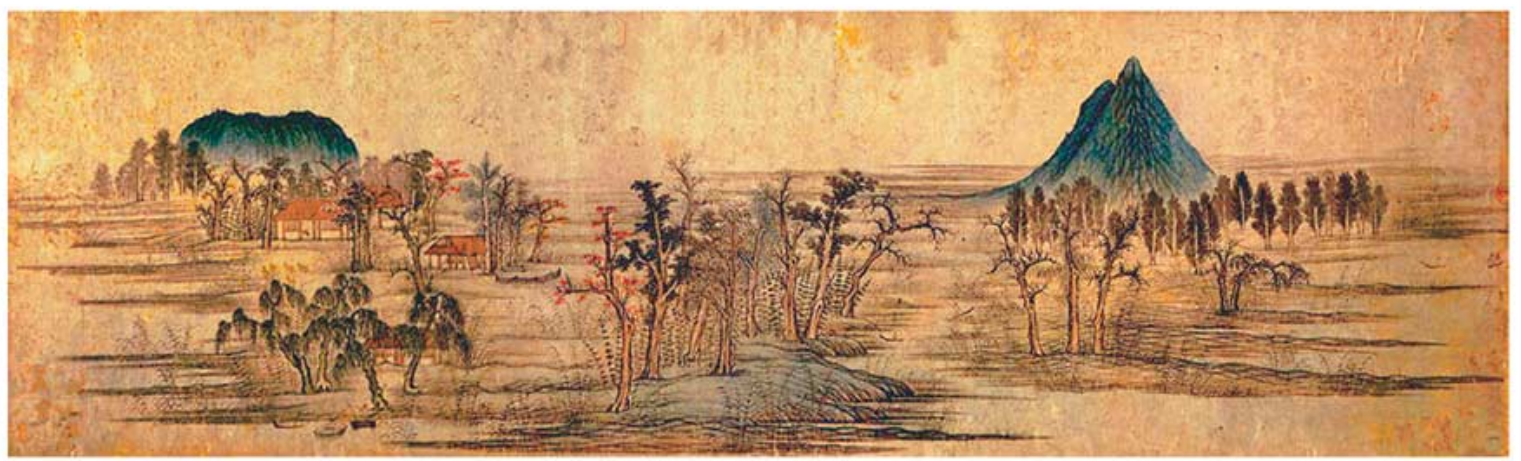

(c)

Fig. 11. (a) Original image of the "Autumn Colors on the Ch'iao and Hua Mountains," (b) enhancement by saturation, and de-saturation $(k=0.5)$; and (c) restored image with words and signets removal from (b).

Fig. 11(c) illustrates the final restored image with the words and signets removed.

\section{B. Lacuna Texture Synthesis}

To demonstrate the proposed algorithm, Figs. 11, 12 and 13 show the experimental results achieved by applying the patching method to specific paintings. The paintings used for the test are "Autumn Colors on the Ch'iao and Hua Mountains," "Maidservant Holding a Duster," and "Three Maidservants Carrying Flower Basins." Clearly these paintings are covered with numerous stains and crevices. The patched regions are marked, and a size $5 \times 5$ neighborhood is applied. Additionally, to emphasize the discontinuous edge in test images, this study employs the weighted mask, in which the ratio of the boundary pixel to the outbound pixel is 20:1.

The three experimental results clearly reveal that the technique successfully patches the images to approximate their original uncorrupt state. Fig. 11 shows how words and signets on the paintings are erased. This technique is designed to remove damages caused by previous owners of the works; for example the Ch'ing Dynasty Emperor Ch'ien Lung liked to write poems and stamp signets on paintings in his collection, destroying the style of the original painting. The proposed 


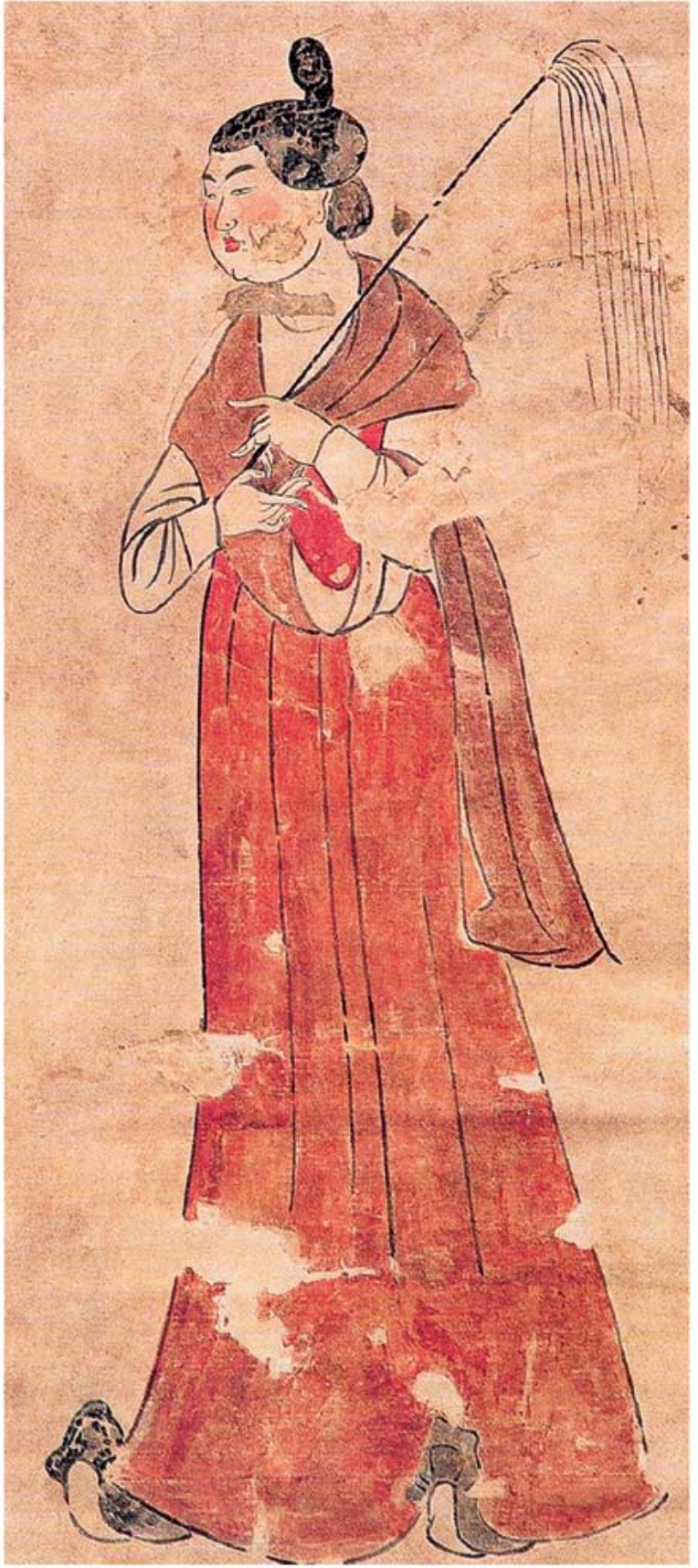

(a)

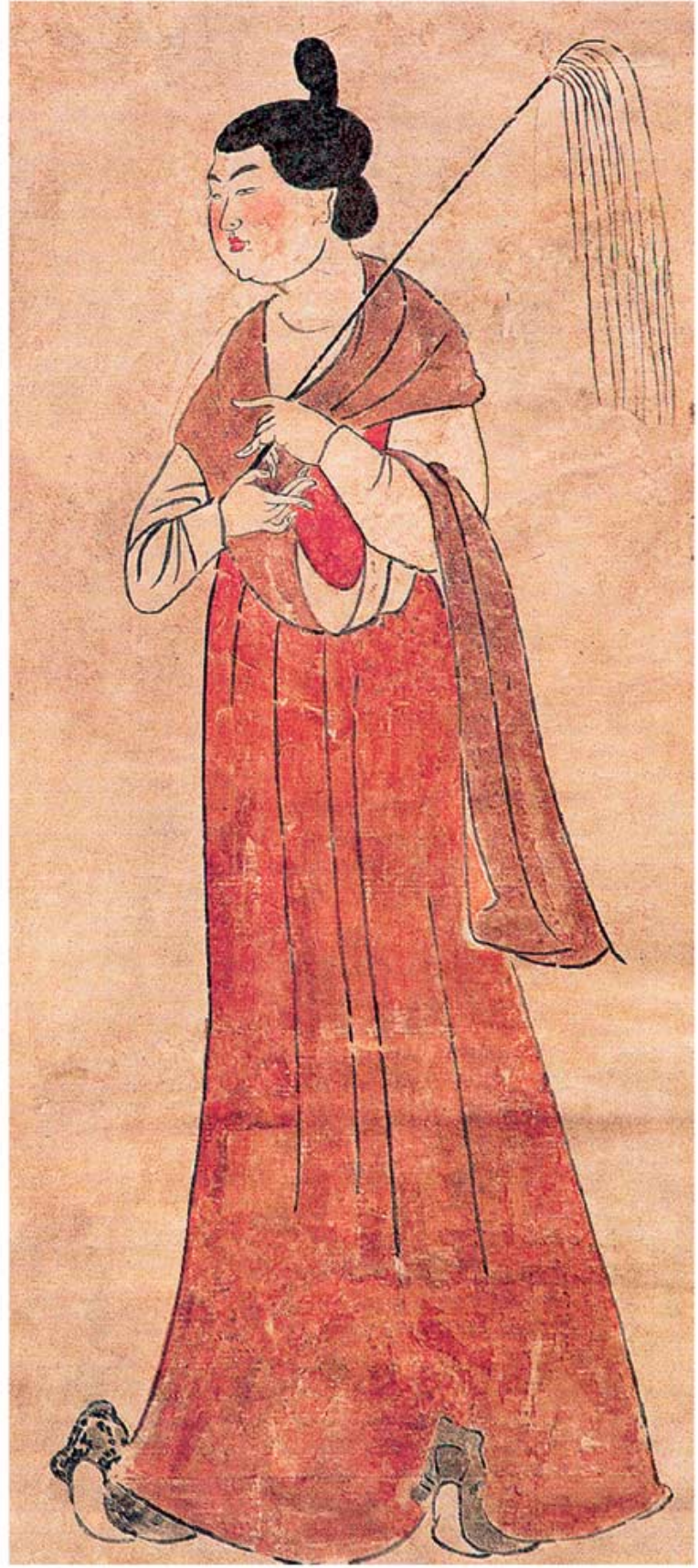

(b)

Fig. 12. (a) Original image of the "Maidservant Holding a Duster" and (b) patched image with stains removal and crevice filling.

algorithm treats words and signets as stains and erases them. Fig. 11(c) shows the restored image after the removal of words and signets.

In Fig. 12, the patched regions involve the hair, cheek, duster, skirt, shawl covered arm, and so on of the maidservant. Notably, several crevices are placed at the boundary of the skirt. Consequently, the incomplete skirt shape is ascribed to discontinuous edges. The experimental result demonstrates that the crevices are not only patched, but actually imitates a pseudo boundary to achieve a complete shape. Fig. 13 displays the results of another experiment, in which the stains are removed from the corrupt image and the image is patched up.

\section{DiscusSIONS}

Besides the proposed method, this study also experiments with two conventional synthesizing algorithms, namely the Wei and Efros' methods. The performance of these three methods is compared by focusing on two examinations, namely the patching capability for edge connection, and the scanning order for neighborhood searching. Subsequently, the influence of auxiliary and sample source is discussed.

\section{A. Patching Capability for Edge Connection}

A difficult problem is whether or not the different synthesizing methods can connect the breach. It is illustrated the 


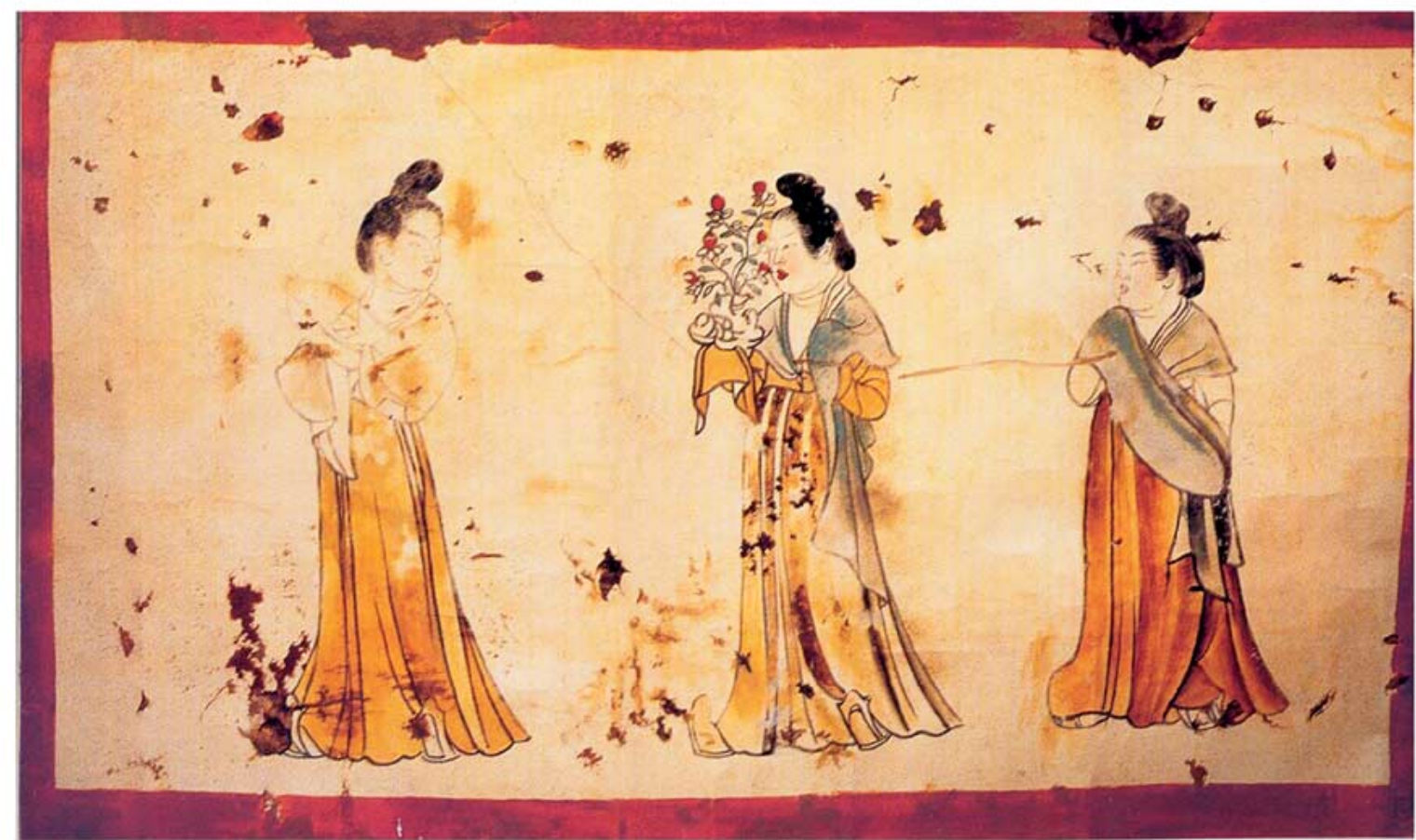

(a)

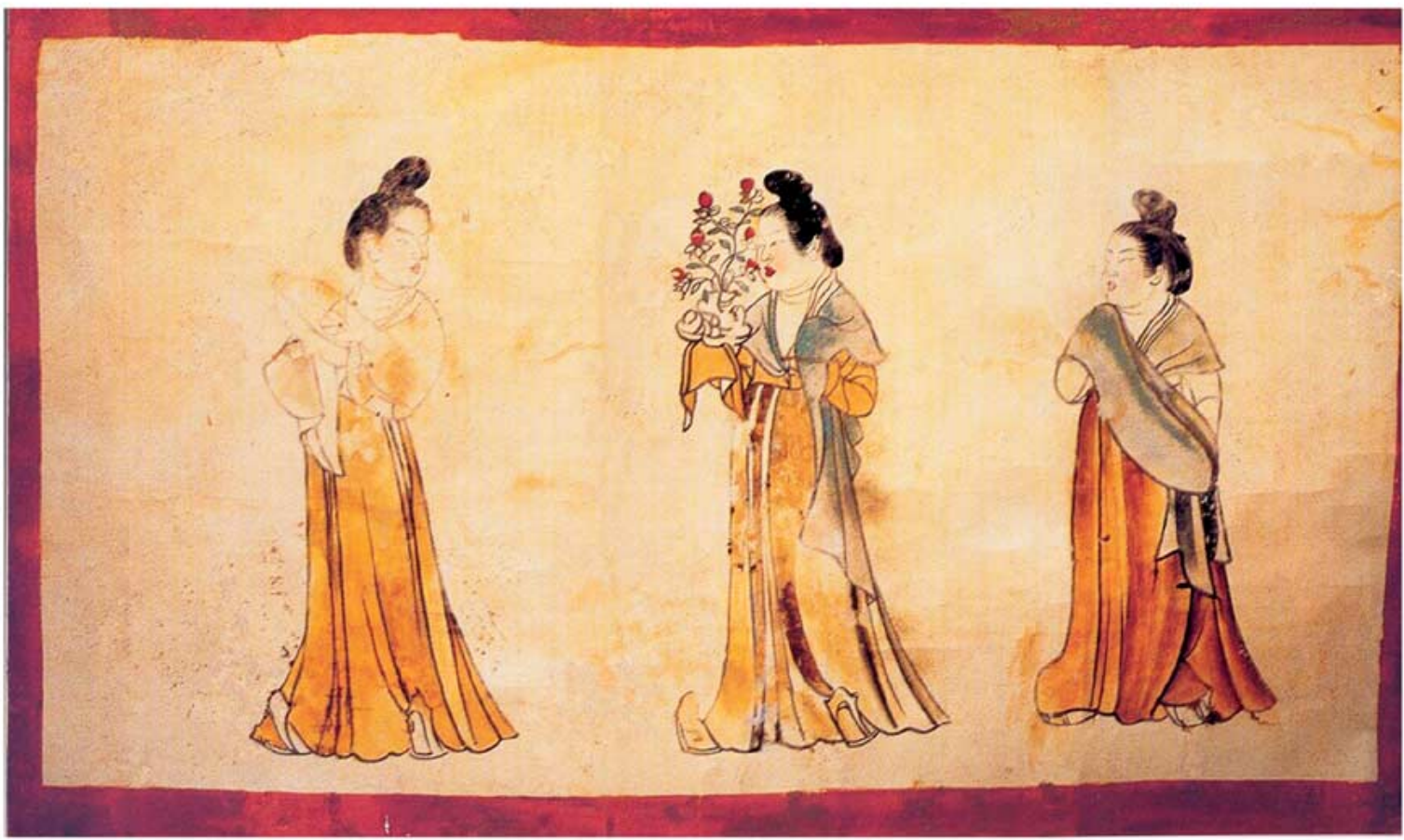

(b)

Fig. 13. (a) Original Image of "Three Maidservants Carrying Flower Basins" and (b) patched image with stains removal.

breach of edge in an image, which is one part of a skirt from the lower-right position of image in Fig. 12(a). This work uses a width of nine pixels to construct two types of neighborhoods, an $L$-shape neighborhood and square neighborhood. The $L$-shape neighborhood, created with Wei's method, consists of 40 pixels, while the square neighborhood, created using Efros and the present method, consists 81 pixels. Additionally, this study assigns the weighted mask with the ratio of boundary to outbound pixels as 20:1.

Clearly, the Efros' method cannot connect the edge in Fig. 14(b); however, both the approach of Wei and that developed here can successfully patch the crevice and then link the two ends of the broken edge, as illustrated in Figs. 14(c) and 14(d), respectively. However, it reveals some clear flaws 


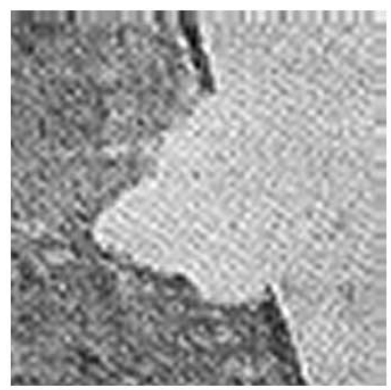

(a)

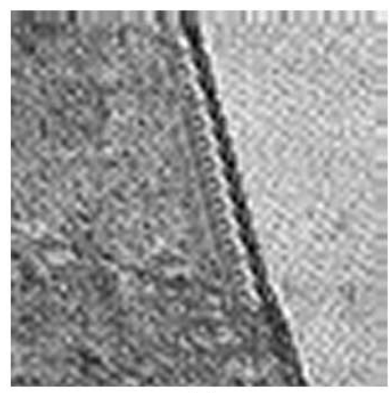

(c)

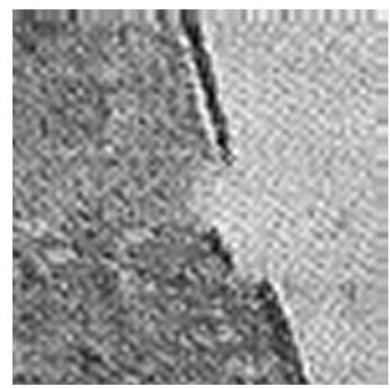

(b)

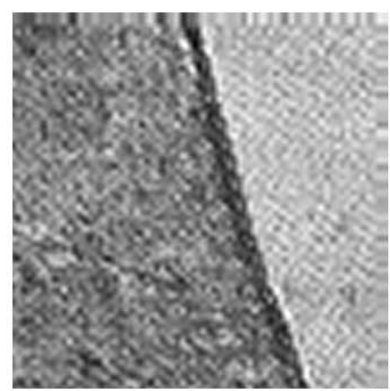

(d)

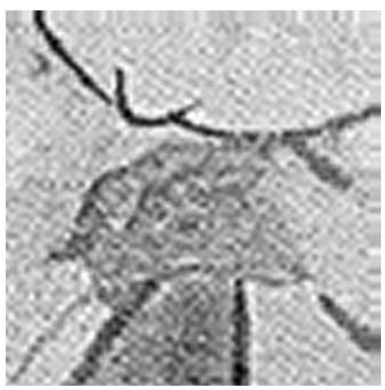

(a)

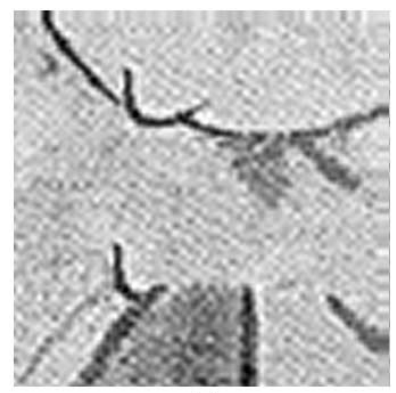

(c)

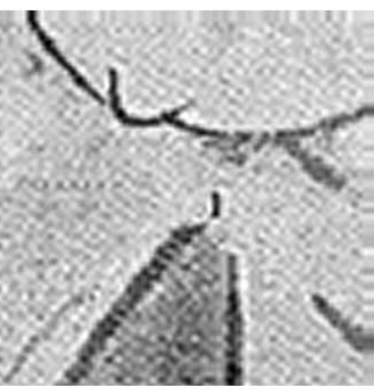

(b)

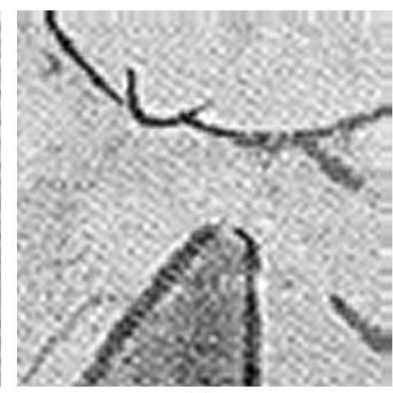

(d)
Fig. 14. An example of the boundary connection. These are (a) corrupt image (b) Efros', (b) Wei's, and (c) our experimental results.

in Fig. 14(c); specifically, some patterns are duplicated on the left side of the black-line edge. Fig. 14(d) illustrates that the proposed approach achieves good results, simultaneously patching the crevice and connecting the edge.

\section{B. Scanning Order for Neighborhood Searching}

The scanning order is discussed, and is divided into two types, namely annular scan and raster scan, are applied to each of the three methods. The approach of Efros and the neighborhood searching strategy developed here employ annular scan, while Wei uses the raster scan.

Fig. 15(a) reveals a flawed region in the image, specifically a part of the shoulder on the upper-middle area of the image in Fig. 12(a). Notably, this flawed region involves three types of pattern, namely the brown colored shawl, skin, and background. The synthesis process aims not only to eliminate undesirable patterns, but also patches the shawl to create a closed shape. Fig. 15(c) shows the result achieved using Wei method, and demonstrates that the shawl on the shoulder is unable to keep the complete shape, and produce the extra pattern simultaneously. In contrast, the approach of Efros and that developed here efficiently synthesize the shawl and patch the flawed region, as shown in Figs. 15(b) and 15(d), respectively.

To analyze the raster scanning order in Wei's method, it starts from left to right and then top to bottom. The antecedent pixels around the central synthesized pixel are applied to measure the distance between two neighborhoods. In Fig. 15(a), the antecedent patterns of the shawl are skin and background, explaining why one part of the flawed region on the shawl is removed and substituted by the color pattern of the skin and background.
Fig. 15. An example of the influence on various scan method. These are (a) corrupt image, (b) Efros', (c) Wei's, and (d) our experimental results.

For annular scanning order, it synthesizes the outside pixels of the patched region first and then gradually progresses inwards. The experimental result obtained using the Efros method contains an extra line compared to the technique developed here, and the edge closeness of the shawl also is worse. The difference between the two methods is that we additionally use the weighted mask, which assigns a larger weighted ratio to the boundary pixel in the neighborhood, to increases the capacity to distinguish pixel characteristic.

\section{The Influence of Auxiliary and Sample Source}

Fig. 16(a) shows a badly damaged example. In the synthesizing experiment, a width of 5 pixels is used to construct the square neighborhood, which contains 25 pixels. Furthermore, the weighted ratio of boundary to outbound pixels is 20:1. Fig. 16(b) and (c) illustrate the patched region and result, respectively.

Fig. 16(c) is a faulty result, and the corrupt region is sufficiently large to cover various textures, such as arms, sleeves, shawl, clothes, background, and so on. This study first applies the auxiliaries to increase the probability of successful synthesis. The auxiliaries draw some one-pixel width lines separating the arm and background, shawl and arm, shawl and sleeve, and sleeve and clothes, respectively, as shown in Fig. 17(a). Subsequently, the flawed region is analyzed and divided into several sub-regions, and then the sample is allocated to each sub-region manually.

In the experiment, three different samples are assigned to clothes, arm (or sleeve), and shawl, as shown in Fig. 17(c), Fig. 17(d) illustrates each of the samples corresponding to the selected region. The original larger sample has size $200 \times 200$, 


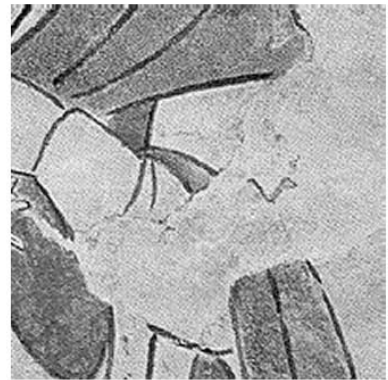

(a)

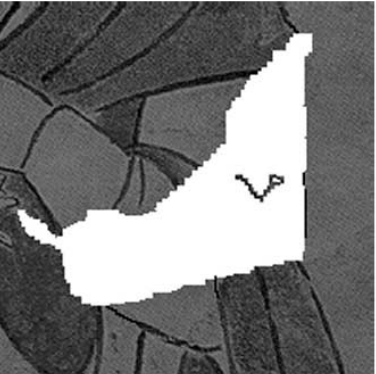

(b)

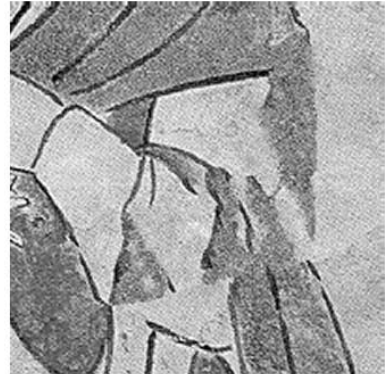

(c)

Fig. 16. Faulty experiment for texture patching. (a) The corrupt image is from Fig. 12(a), (b) patched region, and (c) faulty patched result.

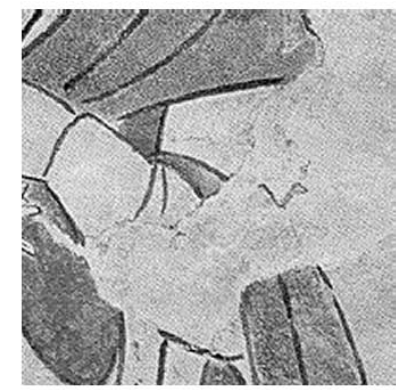

(a)

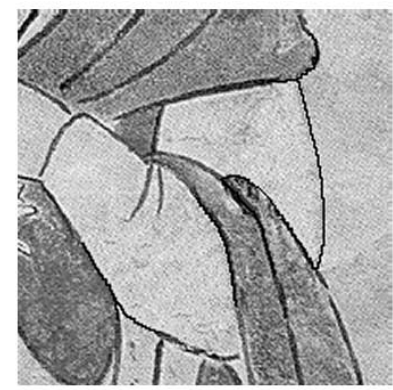

(b)
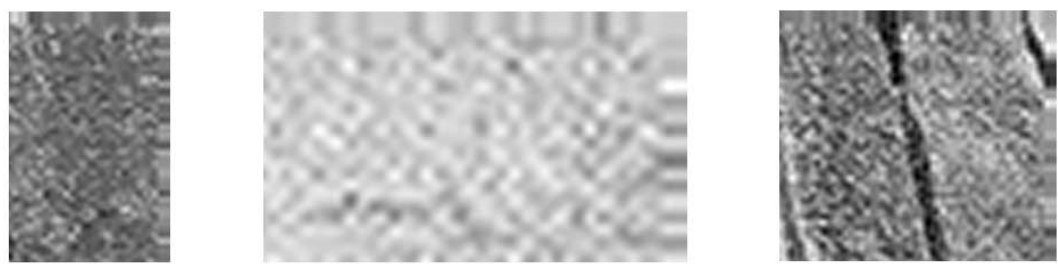

(c)
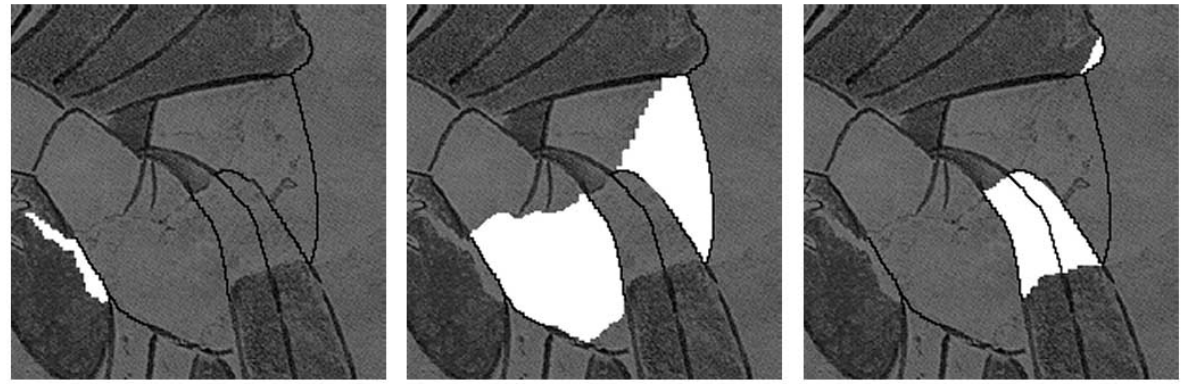

(d)

Fig. 17. Experiment is combined with auxiliary and several smaller sub-region samples. (a) The image is from Fig. 12(a), (b) synthesized result, and (c) three smaller texture samples are to be synthesized in each patched sub-regions in (d).

while the three smaller ones (clothes, arm, and shawl) have size $31 \times 48,39 \times 23$, and $51 \times 46$. Therefore, the smaller samples total $12 \%$ of the larger one, effectively accelerate the neighborhood searching by reducing the search area. Following the above procedure, Fig. 17(b) illustrates the synthesized result, which is better than that illustrated in Fig. 16(c).

\section{CONCLUSIONS}

This study first presents a hybrid method, including brightness and color contrast enhancements. Specifically, the method adopts the $\mathrm{u}^{\prime} \mathrm{v}^{\prime}$ chromaticity domain of the $\mathrm{L} \mathrm{u}^{\prime} \mathrm{v}^{\prime}$ color space, and the Y component of the XYZ color space. The chromatic enhancement includes saturation and de-saturation operations in the $\mathrm{u}^{\prime} \mathrm{v}^{\prime}$ chromaticity diagram. Moreover, adaptive histogram equalization is used to enhance brightness in the $\mathrm{Y}$ component.

Additionally, three approaches, weighted mask, annular scan, and auxiliary, are integrated with synthesization procedure to restore the various aged damages. These methods can eliminate undesirable patterns successfully, and create a seamless boundary between original and synthesized textures. 
From the above experiments and discussions, those demonstrate that the proposed algorithm performs the fine restoring results simply and efficiently, and is superior to previous works. However, an inherent high computation problem exists in the MRF-based neighborhood searching. For example, a 612 $\times 1396$ sized image, shown in Fig. 12(b), approximately 40 minutes is required to synthesize 50 flawed sub-images on a PC with Intel Pentium-III $750 \mathrm{MHz}$ CPU. Further research is needed to reduce these high computation costs.

\section{REFERENCES}

[1] W. K.William. K. Pratt, Digital Image Processing, 2nd ed. New York: Wiley, 2001.

[2] R. C.Rafael C. Gonzalez and R. E.Richard E. Woods, Digital Image Processing. Norwell, MA: Addison-Wesley, 1992.

[3] R. W. G. Hunt, Measuring Color, 2nd ed. Chichester, U.K.: Ellis Horwood, 1987.

[4] L. Lucchese and S. K. Mitra, "Filtering color images in the xyY color space," in Proc. Int. Conf. Image Processing (ICIP 2000), vol. 3, Vancouver, BC, Canada, Sept. 2000, pp. 500-503.

[5] L. Lucchese, S. K. Mitra, and J. Mukherjee, "A new algorithm based on saturation and desaturation in the xy chromaticity diagram for enhancement and re-rendition of color images," in Proc. Int. Conf. Image Processing (ICIP 2001), vol. 2, Thessaloniki, Greece, Sept. 2001, pp. 1077-1080

[6] S. M. Pizer, E. P. Amburn, J. D. Austin, R. Cromartie, A. Geselowitz, T. Greer, B. ter Har Romeny, J. B. Zimmerman, and K. Zuiderveld, "Adaptive histogram equalization and its variations," Comput. Vis., Graph., Image Process., vol. 39, no. 3, pp. 355-368, Sept. 1987.

[7] A. Efros and T. K. Leung, "Texture synthesis by nonparametric sampling," in IEEE Int. Conf. Computer Vision, vol. 2, Sept. 1999, pp. 1033-1038.

[8] L.-Y. Wei and M. Levoy, "Fast texture synthesis using tree-structured vector quantization," in ACM SIGGRAPH'O0 Conf. Proc., 2000, pp. 479-488.

[9] D. J. Heeger and J. R. Bergen, "Pyramid-based texture analysis/synthesis," in ACM SIGGRAPH'95 Conf. Proc., Aug. 1995, pp. 229-238.

[10] J. S. De Bonet, "Multiresolution sampling procedure for analysis and synthesis of texture images," in ACM SIGGRAPH'97 Conf. Proc., Aug. 1997, pp. 361-368.

[11] S. Brooks and N. Dodgson, "Self-similarity based texture editing," in ACM SIGGRAPH'02 Conf. Proc., July 2002, pp. 653-656.

[12] C.-D. Tang, Magnificent Frescos From the Great Tang Dynasty. Shanghai, China: Shaonxi, 1996

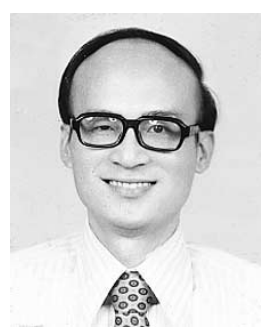

Soo-Chang Pei (M'86-SM'89-F'99) was born in Soo-Auo, Taiwan in 1949. He received B.S.E.E. degree from National Taiwan University (NTU), Taipei, in 1970 and the M.S.E.E. and Ph.D. degrees from the University of California, Santa Barbara (UCSB), in 1972 and 1975, respectively.

$\mathrm{He}$ was an engineering officer in the Chinese Navy Shipyard from 1970 to 1971 . From 1971 to 1975, he was a Research Assistant at UCSB. He was the Professor and Chairman in the Electrical Engineering Department, Tatung Institute of Technology and NTU from 1981 to 1983 and 1995 to 1998, respectively. Presently, he is the Professor and Dean with the Electrical Engineering Department, College of Electrical Engineering and Computer Engineering at NTU. His research interests include digital signal processing, image processing, optical information processing, and laser holography.

Dr. Pei received the National Sun Yet-Sen Academic Achievement Award in Engineering in 1984, the Distinguished Research Award from the National Science Council, R.O.C., from 1990 to 1998, the Outstanding Electrical Engineering Professor Award from the Chinese Institute of Electrical Engineering in 1998, the Academic Achievement Award in Engineering from the Ministry of Education in 1998, the IEEE Fellow in 2000 for contributions to the development of digital eigenfilter design, color image coding and signal compression, and to electrical engineering education in Taiwan, the Pan Wen-Yuan Distinguished Research Award in 2002; and the National Chair Professor Award from Ministry of Education in 2002. He has been President of the Chinese Image Processing and Pattern Recognition Society in Taiwan from 1996-1998, and is a member of Eta Kappa Nu and the Optical Society ofAmerica.

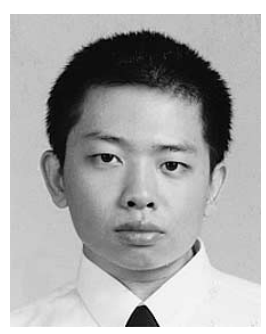

Yi-Chong Zeng received the B.S.E.E. degree from Chung Yuan Christian University (CYCU), Chungli, Taiwan, R.O.C., in 1998 and M.S. degree in Communication Engineering ffom National Taiwan University (NTU), Taipei, Taiwan, R.O.C., in 2000. Currently he is pursuing the Ph.D. degree in communication engineering at NTU.

His research interests include image processing, computer vision, and multimedia application.

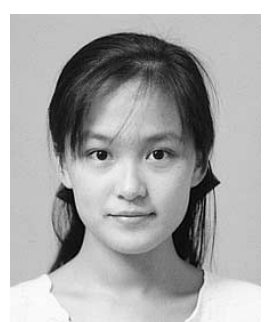

Ching-Hua Chang received the B.S.M.E and B.S.E.E degrees from National Central University, Chungli, Taiwan, R.O.C., in 2001 and the M.S degree in communication Engineering from National Taiwan University, Taipei, Taiwan, in 2003.

She is currently an Engineer with the Realtek Semiconductor Corporation, Hsinchu, Taiwan. Her research interests include image enhancement and color interpolation. 